\title{
MedienPädagogik
}

Zeitschrift für Theorie und Praxis der Medienbildung

\section{Die didaktische und technologische Konzeption der Online-Bildungsplattform für die Bundesinitiative „Jugend ans Netz“}

\section{Christian Swertz}

\section{Die Bundesinitiative Jugend ans Netz}

Die Bundesinitiative Jugend ans Netz (BIJaN) ist ein Forschungs- und Entwicklungsprojekt, das vom Bundesministerium für Familie, Senioren, Frauen und Jugend gefördert wird. Ziel des Projektes ist die Ausstattung von Jugendhilfeeinrichtungen mit Computertechnik durch Public-Privat-Partnership analog zum Projekt Schulen ans Netz. Die Ausstattungsinitiative wird verbunden mit der Entwicklung einer Internetplattform, die Angebote für Jugendliche bereitstellt. In dieser Arbeit steht die pädagogische Konzeption der Internetplattform im Mittelpunkt. Es wird ein Vorschlag für eine didaktische Ontologie sozialer Arbeit zur Diskussion gestellt. Der Vorschlag greift auf die Ergebnisse des L-3-Projekts zurück, in dem eine didaktische Ontologie für das Online-Lernen im Weiterbildungsbereich entwickelt wurde.

Nach welchen Prinzipien kann die geplante Plattform pädagogisch strukturiert werden? Um diese Frage beantworten zu können, ist ein interdisziplinäres Vorgehen erforderlich. Medienpädagogische, didaktische und sozialpädagogische Konzepte müssen verbunden werden. Das kann nur vor dem Hintergrund allgemeinpädagogischer Überlegungen gelingen, die eine integrierende Strukturierung anleiten. Die folgenden Überlegungen stellen zwei Thesen in den Mittelpunkt:

- Computertechnologie erfordert wie jedes Medium eigene Formen der Organisation von Wissen. Die These ist, dass Computertechnologie individuell navigierbare Hypertexte aus bildschirmgroßen Objekten erfordert, die zur Unterstützung von Bildungsprozessen didaktisch strukturiert werden müssen.

- Computertechnologie wird in pädagogischen Handlungsfeldern meist mit einer bestimmten Software eingesetzt. Die Software (Algorithmus) kann innerhalb der Grenzen der Computertechnologie gestaltet werden. Die These ist, dass die Software und damit das Medium nach pädagogischen Gesichtspunkten zu gestalten ist. Mediengestaltung meint dabei die Gestaltung des Mediums, nicht die der Inhalte.

Die erste These erfordert methodisch eine Analyse der Computertechnologie. Eine solche Analyse bedarf einer begrifflichen Grundlage, die es erlaubt, von einzelnen Medien zu abstrahieren. Ein allgemeiner Medien- 
begriff ist erforderlich, da ein klassifikatorischer Medienbegriff angesichts der fortlaufenden Neuerfindung von medialen Formen mit der Computertechnologie keine tragfähige Grundlage für die medienpädagogische Theoriebildung sein kann. Die geplante Mediengestaltung der Computertechnologie bedarf des Medienbegriffs schon wegen der fehlenden etablierten kulturellen Formen, die eine Mediengestaltung orientieren können. Im Folgenden wird ein Ansatz zur Mediengestaltung entwickelt. Methodisch wird dazu vor bildungstheoretischem Hintergrund eine Analyse der medialen Eigenschaften der Computertechnologie mit didaktischen Modellen verbunden.

Hier sind also zunächst die medienpädagogischen, didaktischen und bildungstheoretischen Ansätze (ergänzt um informationswissenschaftliche Konzepte) darzustellen, die der weiteren Argumentation als Folie dienen. Vor diesem Hintergrund wird die didaktische Konzeption und das entwickelte Metadatensystem vorgestellt und Ansätze der technischen Umsetzung skizziert.

\section{Theoretische Grundlagen}

Die Absicht, einen allgemeinen Medienbegriff zu entwickeln, stößt auf ein paradigmatisches Problem: Die Formulierung eines allgemeinen Medienbegriffs ist notwendig auf den Gebrauch eines Mediums angewiesen. Medien sind in Medientheorie und wissenschaftlicher Praxis gleichzeitig präsent. Damit stellt sich die Frage, inwiefern es methodisch möglich ist, einen Begriff von Medien zu entwickeln.

Das Problem mag vernachlässigbar erscheinen, wenn angenommen wird, dass Medien der Theorie prinzipiell neutral gegenüber stehen, dass es also keine Wechselwirkung von Medien und Theoriebildung gibt. Arbeiten aus unterschiedlichen Perspektiven haben jedoch gezeigt, dass die Art und Weise, wie wir uns zu uns selbst, zu anderen Menschen und zur Welt in Beziehung setzen nicht unabhängig von den Medien ist, die wir dabei verwenden. Als Beispiel sei hier die Arbeit von Meyer-Drawe (1996) genannt, die darauf aufmerksam gemacht hat, dass wir uns selbst zunehmend in Abgrenzung zu Maschinen als uns selbst verstehen. Daran wird deutlich, dass wir in der Art und Weise, in der wir zu uns ich sagen, nicht unabhängig von den Medien sind, die wir dabei verwenden. ${ }^{1}$

Welchen Bedingungen muss eine Theorie genügen, damit dieses Problem angemessen erfasst werden kann? Zwei Momente sind besonders relevant:

- Der Umstand, dass eine Theorie von Medien immer auch die Verwendung von Medien impliziert, hat zur Folge, dass die Theorie sich in der

1 Das damit aufgeworfene Problem liegt parallel zu dem, dass wissenschaftliche Theorien nicht unabhängig von den gesellschaftlichen Bedingungen und den individuellen Interessen sind., unter denen sie hervorgebracht werden. 
Praxis ihrer Formulierung stets selbst mit in den Blick nehmen muss. Anders gesagt: Die Theorie muss in ihren eigenen Gegenstandsbereich fallen.

- Es kann kein Standpunkt eingenommen werden, der jenseits von Medien liegt. Es ist aber offensichtlich möglich, ein Medium zu gebrauchen und dabei ein anderes zu betrachten. Zwischen Medien bestehen Beziehungen, oder besser: Relationen, die eine Analyse von Medien ermöglichen, die jedoch keinen absoluten Standpunkt zur Verfügung stellen. Die Theorie muss also relational verfasst sein.

Eine Theorie, die eine solche Methode entwickelt, ist die Allgemeine Pädagogik von Hönigswald. Hönigswald untersucht die Bedingung der Möglichkeit der Tradierung von Geltung als Sinn des pädagogischen Verhaltens (Hönigswald 1927, S. 25). Tradierung von Geltung ist aber auch eine Bedingung der Möglichkeit der wissenschaftlichen Tätigkeit (a.a.O., S. 23). Damit ist die wissenschaftliche Untersuchung des pädagogischen Handelns zugleich auch pädagogisches Handeln. ${ }^{2}$ Hönigswald kann so ansetzen, weil für ihn Prinzip und Faktum zusammenfallen (Meder 1975). Werden Prinzip und Faktum als grundsätzliche Einheit gedacht, liegt die Theorie in ihrem eigenen Gegenstandsbereich (Schneider 1989:, S. 33).

Hönigswald verwendet Relationen als Denkmethode in seiner Analyse pädagogischen Handelns (Schneider 1989, S. 29f.; Meder 1975, S. 9f.). Er denkt Begriffe nicht als unabhängige Ideen, sondern im historischen Wandel (zeitlich) und in Relationen zueinander (räumlich). Damit lenkt er den Blick auf die räumliche und zeitliche Dimension des Wissens. Die räumliche Dimension ist hier als mediale Repräsentation zu kennzeichnen, die zeitliche als Mitteilung und Aneignung. Hönigswalds Ansatz liefert damit den geeigneten Rahmen für eine Medientheorie. Die Bezüge der Hönigswaldschen Konzeption zum Medienbegriff können hier jedoch nicht dicht entwickelt werden und werden daher bei bei folgenden Vorstellung des Medienbegriffs nicht expliziert.

\subsection{Medientheoretische Grundlagen}

Medien sind Gegenstände, die von Menschen zu Zeichen gemacht werden (Abb. 1). Die physikalische, die pragmatische und die semiotische Dimension von Medien sind in dialektischen Relationen zueinander zu denken (Swertz 2001). In der Schriftsprache ist z.B. zwischen der Bedeutung von Wörtern (semiotische Dimension), Farbe und Papier (physikalische Dimension) und dem Verstehen der Bedeutung der Farbe auf dem Papier als Zeichen (pragmatische Dimension) zu unterscheiden.

2 Dieser Umstand zeigt sich darin, dass eine wissenschaftliche Theorie den Anspruch erheben muß, tradiert zu werden. Insofern weist jede wissenschaftliche Theorie pädagogische Momente auf. 


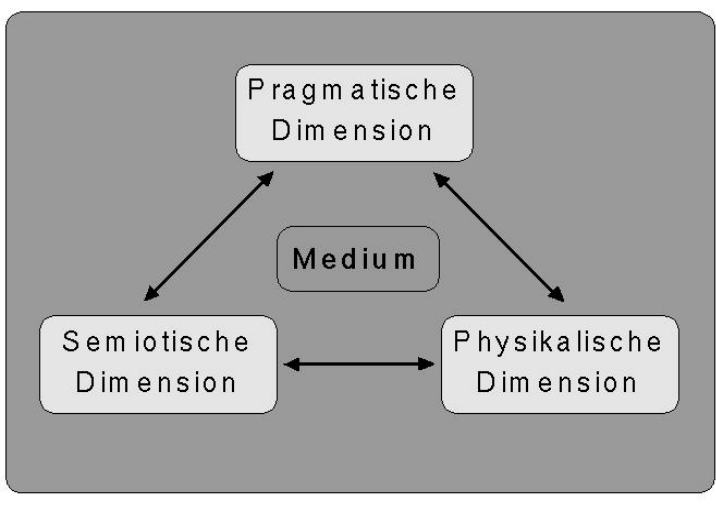

Abb. 1: Möglichkeitsraum der Sätze. Dennoch spiegeln sich in dem Wort die semiotischen Eigenschaften der Sätze. Zuletzt spiegelt sich in der semiotischen Dimension die physikalische Dimension, was z.B. daran sichtbar wird, dass ein gesprochenes Wort geschrieben eine andere zeitliche Struktur aufweist: es ist dauerhafter.

Durch den Austausch einer Dimension bei Beibehaltung einer anderen entsteht Reflexivität zwischen Medien. Insbesondere die Reflexion der Computertechnologie auf andere Medien und die damit verbundenen Verschiebungen in der physikalischen Dimension wird mit der Verwendung von Computertechnik in Bildungsprozessen pädagogisch relevant. Dabei besteht eine Besonderheit: Mit Computertechnologie als elektrischem, digitalen universellen Turingautomaten können zahlreiche Medien simuliert werden. Computertechnologie simuliert ein anderes Medium, indem ein Algorithmus so verwendet wird, als ob es sich um die physikalische Dimension eines Mediums handelt. Der verwendete Algorithmus ist in diesem Fall als physikalische Dimension der Simulation anzusehen, da es für die Computertechnologie keinen Unterschied zwischen der semiotischen und der physikalischen Dimension gibt.

Mit Computern ist es möglich, Wissen in unterschiedlichen medialen Simulationen zu präsentieren. Mit der Simulation sind Veränderungen in der Struktur des Mediums verbunden: die Speicherdauer wird verkürzt und damit die Dauerhaftigkeit gesenkt; die Übermittlungsgeschwindigkeit im Raum steigt. Ein solches Medium forciert, wie in Anlehnung an McLuhan (1992) und Innis (1997) gezeigt werden kann, die Tradierung nichtmystischer Wissensformen und unverbindliche Sozialbeziehungen (Swertz 2001).

Wissen, das an ein elektrisch-digitales Medium gebunden wird, wird beweglicher. Dieser Effekt wird durch die Möglichkeit, Wissen durch Algorithmen zu bearbeiten, verstärkt. Dieses dynamisierende Moment der Computertechnologie forciert die Individualisierung in der Aneignung von Wissensbeständen. In die gleiche Richtung wirkt

3 Wie Meder schreibt, operiert jede Reflexion auf einer Metaebene über einer Objektebene. So reflektiert z.B. Sprache auf die Musik (Meder 1995a, S. 14). Die Operation auf der Metaebene erfaßt die Objektebene dabei nicht vollständig, sondern betont einen Aspekt, der dabei überhöht wird, indem er die Objektebene gleichsam spiegelt. Die damit verbundene Auswahl wird hier stärker in den Mittelpunkt gerückt. 
die im Vergleich zum Buchdruck geringe Auflösung von Bildschirmen. Die geringe Auflösung macht eine sinnliche Ergänzung der Wahrnehmung erforderlich (McLuhan 1992). Die fehlenden Details müssen vervollständigt werden. Dadurch forcieren Bildschirme involvierende Beziehungen - im Gegensatz zur distanzierenden Wirkung des Buchdrucks.

Neben der geringen Auflösung verändern Bildschirme die sinnliche Wahrnehmung von Kontexten. Während bei einem Buch der ganze Text wenigstens haptisch erfahrbar ist, entzieht sich die elektrische Repräsentation im Computerspeicher der Wahrnehmung. Wegen dieser Eigenschaft hat Meder Bildschirme als Schlüssellöcher bezeichnet (Meder 1998, S. 34f.). Die Darstellung hat keinen abschattierten Hintergrund, der als Kontext von Zeichen wahrgenommen und interpretiert werden könnte. Für die mediendidaktische Gestaltung von computerbasierten Wissenssystemen ist es daher erforderlich, von einer Bildschirmseite als kleinster zusammenhängender Einheit auszugehen. Der Kontext einer Bildschirmseite muss zusätzlich auf dem Bildschirm dargestellt werden, um den Hintergrund der Zeichen sinnlich erfahrbar zu machen. Der Kontext muss durch ein Navigationskonzept erschlossen werden, mit dem der jeweilige Ausschnitt in Beziehung zum vorhandenen Wissen gesetzt wird; damit wird zugleich „Häppchenwissen“ vermieden.

Diese Eigenschaften der Computertechnik passen zum Fernsehen. Computertechnologie ist daher ein angemessenes Medium für ein Publikum, für das Fernsehen das dominante Medium darstellt. Für ein solches Publikum ist Computertechnologie als Medium der Bildung zu empfehlen; nicht aber für ein Publikum, für das der Buchdruck das dominante Medium darstellt. Der involvierende Charakter, den das fernsehgewohnte Publikum versteht, zeigt sich z.B. im motivierenden Effekt der Computertechnologie.

Computertechnologie forciert unverbindliche und involvierende Sozialbeziehungen sowie eine Individualisierung in der Auseinandersetzung mit Wissen. Damit deutet sich bereits an, dass die kulturelle Aneignung der Computertechnologie nicht ohne Wirkung auf die Kultur, und damit auch auf unsere Vorstellungen von Bildung bleibt.

\subsection{Bildungstheoretische Grundlagen}

Eine bildungstheoretische Analyse der kulturellen Aneignung von Computertechnologie hat Meder (1998) vorgelegt. Computertechnologie gilt ihm als Kulturtechnik, wenn sie (1) das ganze gesellschaftliche Leben auf allen Ebenen menschlicher Aktivitäten durchdringt und wenn sie (2) zugleich ein gesellschaftliches Problem betrifft, dessen Lösung als ein Wert an sich angesehen wird. 
1) Computertechnologie durchdringt das gesellschaftliche Leben durch die Verbreitung und als abstrakter Problemlösungsautomat, da in der Moderne Welt nur noch als Problem wahrgenommen werden kann und daher überall Computer für Problemlösungen verwendet werden können.

2) Die Lösung der Probleme wird als Wert an sich angesehen, wenn die Lösung tradiert wird. Tradierung bestimmt Meder mit Hönigswald als Weitergabe von Kulturgütern von einer Generation auf die übernächste vermittels der zeitlich nächsten (Hönigswald 1927: 25). Die Tradierung geschieht in der Moderne im Modus des Widerstreits um Geltung. Computertechnologie ermöglicht diesen Widerstreit um Geltung, weil sie als abstrakter Problemlösungsautomat mit Inhalten gefüllt werden kann.

Computertechnologie als Kulturtechnik kann in Bildungsprozessen verwendet werden. Der Bildungsprozess ist die Herstellung eines individuellen Verhältnisses zur Welt, zur Gesellschaft und zu sich selbst (Meder 1998). Die drei Verhältnisse sind sprachlich vermittelt. Computertechnologie erlaubt es, diese Verhältnisse auf spezifische Weise herauszubilden. Sprachliches Problemlösen wird durch die Erfindung und Erprobung von geeigneten Sprachen ermöglicht. Computertechnologie macht es dabei erforderlich, nicht nur die Sprache, sondern auch die Konstruktionsprinzipien der sprachlichen Verhältnisse zu reflektieren. Das Medium Computer erfordert im Bildungsprozess das Reflexiv-werden der Sprache. Sprache als Mittel der Reflexion des Verhältnisses zur Welt wird damit als Mittel der Reflexion auf dieses Verhältnis verwendet - und damit doppelt reflexiv. Der gebildete Mensch wird so zum Sprachspieler. Nach dieser Analyse von Meder forciert Computertechnologie den Umstand, dass Lernende sich im Lernprozess nicht zur zum Wissen, sondern auch zum Prozess des Lernens in Beziehung setzen. Computertechnologie forciert damit selbstgesteuertes Lernen. ${ }^{4}$

Neben der doppelt reflexiven Struktur wird durch Computertechnologie unser Verhältnis zu anderen ästhetisiert. Selbstdarstellung als Performanz eines kontingenten Entwurfs rückt nach Meder in den Mittelpunkt des Bildungsgedankens. Diese Ästhetisierung findet sich auch in unserem Verhältnis zur Welt. Es geht in computervermittelten Bildungsprozessen um die beste mögliche Darstellung von Welt, nicht die Darstellung der besten Welt. Es geht nicht mehr um Einheit, sondern um die feine Differenz. Der gebildete Mensch als Sprachspieler bewahrt in der feinen Differenz seine Eigenständigkeit auch gegenüber der Gesellschaft. Bei der Konzeption einer Online-Plattform muss daher die ästhetische Selbstdarstellung der Lernenden und die Möglichkeit, dabei Differenzen herzustellen, berücksichtigt werden. Wie muss Wissen organisiert werden, damit diesen Anforderungen angemessen begegnet werden kann?

4 Hier drängt sich die These auf, daß die derzeitigen Tendenzen zum selbstgesteuerten, selbstorganisierten oder informellen Lernen, die diesem Umstand Rechnung tragen, als pädagogische Reaktion auf einen medieninduzierten kulturellen Wandel zu verstehen sind. 


\subsection{Didaktische Grundlagen}

Die Organisation von Wissen ist Gegenstand der Didaktik. Da die Produktion von Online-Lernumgebungen in der Regel mit Computern erfolgt, kann auf Grundlage eines didaktischen Konzepts nicht nur Wissen, sondern auch die Software so gestaltet werden, dass die didaktisch sinnvolle Produktion und Rezeption von Inhalten forciert wird; die Struktur des Mediums setzt dann unpassenden Formen der Wissensorganisation gleichsam einen Widerstand entgegen, wobei Unangemessenheit als Zug im Sprachspiel jederzeit möglich bleibt.

Dabei mag es zunächst überraschen, dass ein Konzept für ein sozialpädagogisches Projekt auf didaktische Überlegungen zurückgeführt wird; zumal die folgenden Überlegungen nicht auf die Vermittlung von sozialpädagogischen Kenntnissen, sondern auf die soziale Arbeit selbst zielen. Wird jedoch als Gegenstand der Didaktik die Organisation von Wissen nach pädagogischen Prinzipien und pädagogisches Handeln als Verständigung über Wissensbestände angesehen, betreffen didaktische Überlegungen pädagogisches Handeln schlechthin.

Die Computertechnologie stellt für die Didaktik eine besondere Herausforderung dar, weil sie Wissensbestände dynamisiert. Die Anordnung von Wissensbeständen kann während des Verständigungsprozesses verändert werden. Während beim Buchdruck die Anordnung vor dem Druck festgelegt wird und dann immer gleiche Exemplare produziert werden, die alle alles auf dieselbe Art und Weise lehren, kann mit Computertechnologie die Anordnung der Inhalte während des Lernprozesses innerhalb der Grenzen universeller Turingautomaten individuell variiert werden. So können feine Differenzen hergestellt werden. Eine Festlegung auf ein bestimmtes didaktisches Modell (z.B. getaktetes Lernen, entdeckendes Lernen etc.) wird durch die dynamisierende und individualisierende Struktur der Computertechnologie jedoch unterlaufen. Die Inhaltswahl kann individuell erfolgen und das verwendete didaktische Modell kann für oder von jeder und jedem Lernenden individuell gewählt werden. ${ }^{5}$

Damit konkretisiert sich die Frage nach der Organisation des Wissens: Wie kann Wissen so organisiert werden, dass eine individuelle Auswahl des Wissens und des didaktischen Modells möglich ist? Die Möglichkeit einer solchen Didaktik hat Flechsig mit seinem Göttinger Katalog demonstriert (Flechsig 1983). Seine Systematik verdichtet die Vielfalt didaktischen Handelns in 20 Arbeitsmodelle. Wissen muss für individuelle Lernverläufe also nicht beliebig reorganisierbar sein, sondern im Blick auf 20 Modelle.

Wie muss Wissen organisiert werden, damit es nach didaktischen Modellen angeordnet werden kann? Dazu ist es sinnvoll, Wissen so zu organisieren, dass nicht jedes Modell einzeln abgebildet wird, sondern dass Wissen,

5 Damit ist nicht verbunden, die Lernenden völlig frei zu setzen. Auf den Ausdruck des Anspruchs auf Tradierung von Geltung kann, wie Höngiswald (1927) gezeigt hat, letztlich nicht verzichtet werden. 
das in verschiedenen Modellen zu unterschiedlichen Zeitpunkten benötigt wird, nur einmal produziert und dann bei Bedarf ausgegeben wird. Die medientheoretischen Überlegungen im letzten Abschnitt haben deutlich gemacht, dass dabei von bildschirmgroßen Wissenseinheiten auszugehen ist. Damit diese Wissenseinheiten in unterschiedliche Sequenzen gebracht werden können müssen sie in sich geschlossen, d.h. kohäsiv sein (Swertz 2000). Diese in sich geschlossenen Wissenseinheiten können dann in das gewünschte didaktische Modell angeordnet werden.

Diese Anordnung läßt sich nun zunächst nicht automatisieren, da für die Anordnung ein Verstehen der Inhalte der Wissenseinheiten erforderlich ist. Für universelle Turingautomaten ist aber kein Verstehen möglich. Um eine automatische Sequenzierung zu ermöglichen, muss die didaktische Bedeutung des Wissens zusätzlich erfasst werden. Dazu werden didaktische Metadatensysteme verwendet. Die didaktische Bedeutung des Wissens wird durch die Autorinnen und Autoren mit Hilfe der Metadaten abgebildet. Die Autorinnen und Autoren verstehen das Wissen und können daher eine zutreffende Typisierung vornehmen. Dabei kann keine letztlich eindeutige Typisierung vorgenommen werden; es gilt, die bildschirmgroßen Wissenseinheiten mit dem dominanten Medien- und Wissenstyp zu typisieren. Das Metadatensystem orientiert dabei zugleich die Inhaltsproduktion und senkt so den Produktionsaufwand.

Mit Computertechnologie kann dann die individuelle Sequenzierung auf Grund der Metadaten automatisiert werden. Auch dabei geht Computertechnologie wieder verständnislos vor. Es muss vorher exakt festgelegt werden, wann welches Wissen nach welchem Modell präsentiert werden soll. Zusätzlich kann die manuelle Sequenzierung durch Lehrende und Lernende offen gehalten werden.

Ein Ansatz für die didaktische Typisierung von bildschirmgroßen Wissenseinheiten findet sich ebenfalls bei Flechsig, der für das CEDID-System (Computerergänztes didaktisches Design) die Unterscheidung von Orientierungswissen, Handlungswissen, Erklärungswissen und Quellenwissen eingeführt hat. Diese Unterteilung umfasst Wissen, das rezeptiv angeeignet wird.

Mit der didaktischen Typisierung und der automatischen Sequenzierung von Wissenseinheiten wird nun bereits eine Anpassung von Lernverläufen an den individuellen Bedarf und damit die Herstellung feiner Differenzen möglich. Es ist aber nicht möglich, den einzig richtigen Lernweg für jede und jeden Lernenden im Vorfeld festzulegen. Da Lernen kein deterministisch bestimmbarer Prozess ist, muss es Lernenden ermöglicht werden, den geplanten Lernweg jederzeit zu verlassen und damit in den Widerstreit zum Wissen zu treten. Das kann zum einen durch die Kooperation mit Tutorinnen und Tutoren geschehen, denen gegenüber das Wissen in Frage gestellt wird, zum anderen durch die freie Navigation in der Wissensbasis. Das Wissen muss also so organisiert werden, dass nicht nur die Navigation anhand didaktischer Modelle, sondern auch die freie Navigation in der Wissensbasis möglich ist. Dazu sind informationswissenschaftliche Verfahren geeignet. 


\subsection{Informationswissenschaftliche Grundlagen}

Während es in der Mediendidaktik darum geht, Wissen mit dem Ziel der Vermittlung medial zu organisieren, geht es in der Informationswissenschaft darum, bestehende Dokumente zu erschließen. Entscheidend für das hier diskutierte Konzept ist dabei der Ansatz, Dokumente so zu erschließen, dass sie bedarfsgerecht an Nachfragerinnen und Nachfrager vermittelt werden können (Seeger 1991, S. 23). Retrievalverfahren ermöglichen es Benutzerinnen und Benutzern, Dokumente selbst zu lokalisieren (Ratzek 1991, S. 242). Im Mittelpunkt steht dabei das Problem, auf eine Suchanfrage hin alle relevanten Dokumente zu finden (recall) und keine irrelevanten Dokumente nachzuweisen (precision). So ist beim wissenschaftlichen Arbeiten die Recherche vorhandener Literatur ein wichtiger Schritt bei der Erschließung eines neuen Themas. Aus didaktischer Sicht Sicht kann diese Tätigkeit als selbstgesteuertes Lernen verstanden werden. Hilfreich sind informationswissenschaftliche Methoden aber auch innerhalb eines formellen Kurses, weil mit diesen Methoden die Lernenden auch unabhängig von geplanten Lernverläufen für sie relevantes Wissen aneignen können.

Welche Erschließungsverfahren sind nun für Lernplattformen geeignet? Informationswissenschaftliche Verfahren erschließen Dokumente formal (Titel, Autor etc.) und inhaltlich. Zur inhaltlichen Erschließung werden neben Abstracts Indexierungsverfahren eingesetzt. Dabei kann zwischen Volltextindexierung als offenes System, bei dem die Indexierungsterme aus den Dokumenten entnommen werden, und Klassifikationen und Thesauri als geschlossene Systeme, die mit kontrollierten Begriffssystemen arbeiten, unterschieden werden (Knorz 1991, S. 126). Klassifikationen stellen ein universelles Begriffssystem dar, mit dem jedes Dokument mit Hilfe der künstlichen Klassifikationssprache erschlossen wird (Dahlberg 1974). Thesauri basieren auf der natürlichen Sprache, die in einem Gebiet verwendet wird. Aus dieser Sprache wird ein kontrolliertes Indexierungs- und Retrievalvokabular entwickelt, in dem die Termini durch Relatoren verbunden sind (Wersig 1978, S. 28ff.).

Für die Zwecke der Lernplattformentwicklung ist zunächst die Volltextindexierung der Wissenseinheiten sinnvoll, die eine hohe Flexibilität bei der Recherche bietet. Zwar steigen mit dem Umfang der Wissensbasis die Anforderungen an die Suchstrategien; im Wissensbestand eines Kurses kann diese Strategie aber sehr effizient sein.

Für den Aufbau einer Lernplattform nach der bisher skizzierten Didaktik stellt sich das Problem, die didaktisch bestimmten Wissenseinheiten thematisch zu ordnen. Es ist naheliegend, alle Wissensarten zu einem Thema zusammenzufassen. Eine solche Zusammenstellung wird als Lerneinheit bezeichnet. Hier wird nun vorgeschlagen, das Thema der Lerneinheit als Indexierungsterm (und nicht als Titel) aufzufassen.

Die Lerneinheiten können mit einer Klassifikation oder einem Thesaurus organisiert werden. Einer Universalklassifikation entspricht in didaktischem Kontext ein in Feinziele operationalisiertes fächerübergreifendes Curriculum. 
Ein solche Curriculum liegt jedoch nicht vor und ist wahrscheinlich auch nicht zu entwickeln. Schon deswegen ist ein Thesaurus vorzuziehen. Die Themen sind in einem Thesaurus einer Bezeichungskontrolle, einer terminologischen Kontrolle (z.B. Homonym- und Polysemkontrolle) und einer Begriffskontrolle zu unterziehen (Wersig 1978, S. 46ff.).

Die Begriffe werden in einem Thesaurus durch typisierte Relationen miteinander verbunden (vgl. z.B. DIN 1483). Mit den Relationen wird die thematische Struktur eines Gebiets abgebildet. Auf dieser Grundlage können den Lernenden Retrieval- und Navigationswerkzeuge angeboten werden (z.B. Schlagwortsuche, Indexe, Wissenslandkarten, Gliederungen). Mit den Relationen werden Wege zwischen den Begriffen erschlossen. Es bietet sich an, diese Wege didaktisch zu verstehen. Auf die Linkstruktur können didaktische Modelle abgebildet werden, die sich an der sachlogischen Struktur des Gebiets orientieren.

Durch die Integration informationswissenschaftlicher Methoden in Online-Lernumgebungen erhalten Lernende und Lehrende die Möglichkeit, benötigtes Wissen frei in der Wissensbasis zu recherchieren. Innerhalb eines Kurses können mit Retrievalverfahren geplante Lernsequenzen durchbrochen werden. Damit können die Lernenden jederzeit die Verantwortung für den Lernprozess übernehmen, d.h. selbstbestimmt Lernen. Es ist anzunehmen, dass diese Verantwortungsübernahme durch die mediale Struktur der Computertechnologie forciert wird. Damit wird die Übernahme als Verantwortung für den eigenen Lernprozess als verstecktes Lernziel in der Struktur der Plattform realisiert. Damit sind die Grundzüge und Hintergründe des hier vorgestellten didaktischen Metadatensystems entwickelt; die Details des Systems können jetzt in den Blick genommen werden.

\section{Das didaktische Metadatensystem}

Der Versuch, die dargestellten theoretischen Grundüberlegungen in ein praktisch anwendbares Metadatensystem umzusetzen, führt notwendig auf das pädagogische Theorie-Praxis-Problem. Da eine Deduktion praktischer Handlungsanweisungen wegen der Professionalisierungsbedürftigkeit pädagogischen Handelns (Oevermann 1996) auch für die Inhaltsproduktion nicht möglich ist, kann das Metadatensystem nur heuristisch entwickelt werden. Das Metadatensystem muss also dem jeweiligen Bedarf angepasst werden. ${ }^{6}$ Bei der Anpassung des Metadatensystems lohnt es sich, Grundkategorien möglichst weitgehend beizubehalten, um das Überspielen von Wissensbeständen von einer Lernplattform zu einer anderen zu erleichtern. In diesem Sinne wird im folgenden die Anpassung des Metadatensystems, das unter der Leitung von Norbert Meder im L-3Projekt für die berufliche Bildung entwickelt worden ist (vgl.

6 Eine andere Vorgehensweise würde auch die historische und kulturelle Relativität der Tradierung von Wissen übergehen. 
dazu Meder: in Druck sowie die L-3-Projektdokumentation) für das BIJaN-Projekt beschrieben. Die Details des Metadatensystems werden dabei vollständig vorgestellt, aus Platzgründen werden aber nur die Veränderungen expliziert. Die wesentlichen Grundzüge (grundlegende Wissensarten, Unterteilung in Wissens- und Lerneinheiten, Relationierung der Lerneinheiten etc.) wurden beibehalten. Anpassungen musste erst unterhalb der dritten Ebene des Metadatensystems vorgenommen werden.

Bisher wurde gezeigt, dass Online-Lernumgebungen aus medienpädagogischer und didaktischer Sicht aus bildschirmgroßen Wissenseinheiten aufgebaut werden müssen, die durch den Medientyp und die didaktischen Wissensart bestimmt sind. Die Wissenseinheiten werden zu Lerneinheiten zusammengefasst, die mit typisierten Relationen verbunden werden.

Für die Typisierung wird dabei in der erstene Ebene zwischen rezeptivem, interaktivem, kooperativem und relationalem Wissen unterschieden:

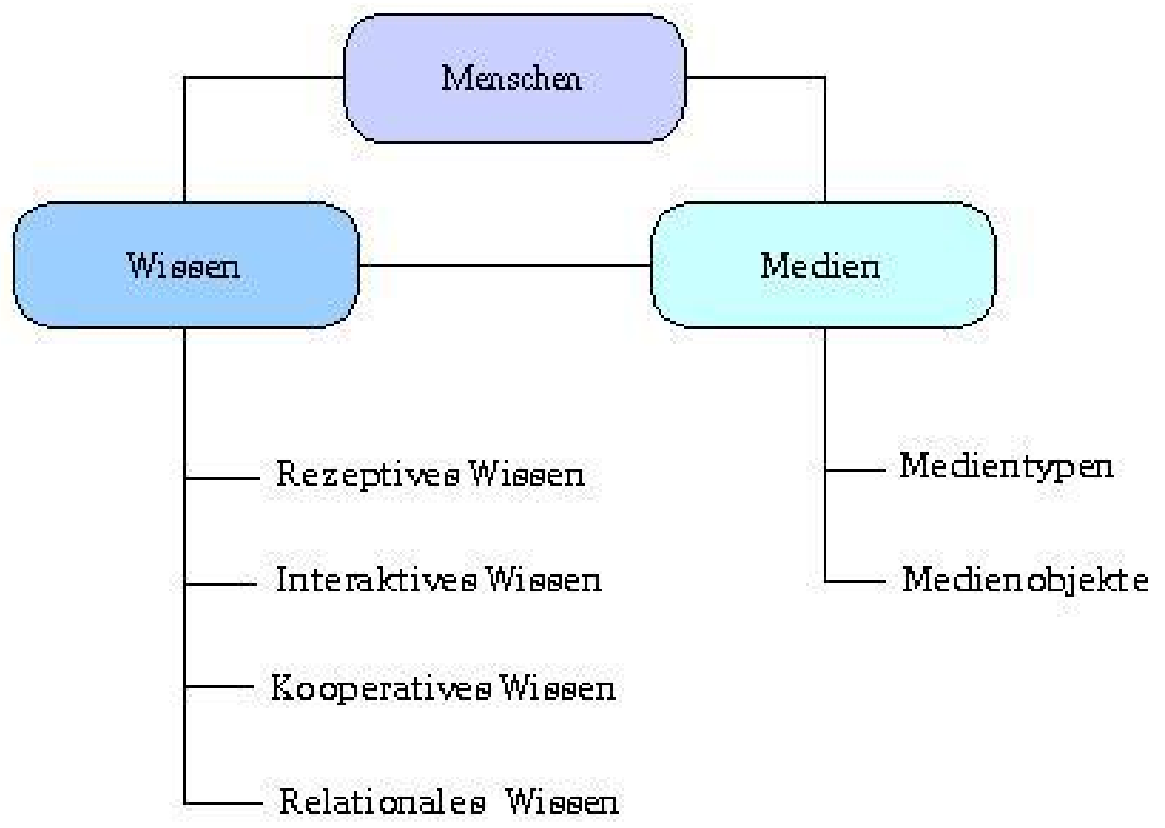

- Rezeptive Wissenseinheiten sind solche, bei denen Wissen passiv wahrgenommen wird.

- Interaktive Wissenseinheiten sind solche, bei denen Lernende in Interaktion mit dem Computer treten.

- Kooperative Wissenseinheiten sind solche, bei denen die Lernenden in Kommunikation mit Lehrenden oder Lernenden treten.

- Relationales Wissen ist solches, bei dem die Lernenden Relationen zwischen Lerneinheiten folgen. können.

Diese Unterscheidung ist auch bei den Medientypen zu berücksichtigen. 


\subsection{Medien}

Eine Wissenseinheit kann durch den Simulationscharakter der Computertechnologie in unterschiedlichen medialen Formaten präsentiert werden. Diese medialen Formate weisen unterschiedliche didaktische Qualitäten auf. Daher muss auch das mediale Format der Wissenseinheiten typisiert werden. Der didaktische Medientyp ist jedoch nicht mit technischen Formaten identifizierbar. Um die technischen Formate erfassen zu können, wurde die didaktische Ontologie für das BIJaN-Projekt gegenüber der L-3- Systematik um Medienobjekte erweitert.

\subsubsection{Medienobjekte}

Medienobjekte enthalten jeweils ein technisch bestimmtes Format für die Eingabe und Darstellung von Wissen. Beispiele sind ein Chat, der im Eingabemodus aufgerufen eine Konfigurationsseite präsentiert und im Darstellungsmodus den erstellten Chatraum, oder eine HTML-Seite, die im Eingabmodus einen WhatYouSeeIsWhatYouGet - Editor präsentiert und im Darstellungsmodus die HTML-Seite ausgibt. Beispiele für die geplanten Medienobjekte für das BIJaN-Projekt sind: HTML-Seite, Grafikseite, HTML-/Grafikseite, Flash-Animation, MPEG2-Video, Linkliste, Dateidownload, Mehrfachwahlfragen, lickertskalierte Fragen, Kursauswahl, Wissenssuche, Benutzerinnen- und Benutzersuche, Fragebogen, Benutzerregistrierung, Benutzerdatenverwaltung, Institutionendaten, Events, Beratersuche, Metadatenkonfiguration, Autorentool, synchrone Lernberatung, asynchrone Lernberatung, synchrone psychosoziale Beratung und asynchrone psychosoziale Betratung. Diese Liste wird im Laufe des Projekts anhand des jeweiligen Bedarfs weiterentwickelt.

Das Konzept der Medienobjekte bietet den Vorteil, dass das System technisch anhand des jeweiligen Bedarfs leicht erweitert werden kann. Die Medienobjekte können durch die Zuordnung zu Wissens- und Lerneinheiten flexibel im System eingesetzt werden. Indem z.B. auch die Kursauswahl als eine Form von Wissen betrachtet wird, wird die Struktur des Systems selbst wieder zum Gegenstand der didaktischen Organisation von Wissen mit dem System; die Lernplattform rückt damit in ihren eigenen Gegenstandsbereich. Die Medienobjekte enthalten nun aus didaktischer Sicht unterschiedliche Medientypen.

\subsubsection{Medientypen}

Die Medientypen werden anhand der grundlegenden Kategorien unterschieden in Kommunikationsmedien, Präsentationsmedien und Interaktionsmedien. In den weiteren Kategorien geht es nicht darum, möglichst differenziert die technisch möglichen Variationen wiederzugeben (dazu werden Medienobjekte verwendet), sondern die Medien anhand der didaktischen Eigenschaften zu typisieren. Die Kategorien der Präsentationsmedien erlauben auch Variationen 
des Abstraktionsgrads. Unterscheidet man zwischen bildlicher, ikonischer und symbolischer Darstellung, ist klar, dass die bildliche Darstellung in einem Foto, die ikonische in einer Grafik und die symbolische in einem Text zu finden ist.

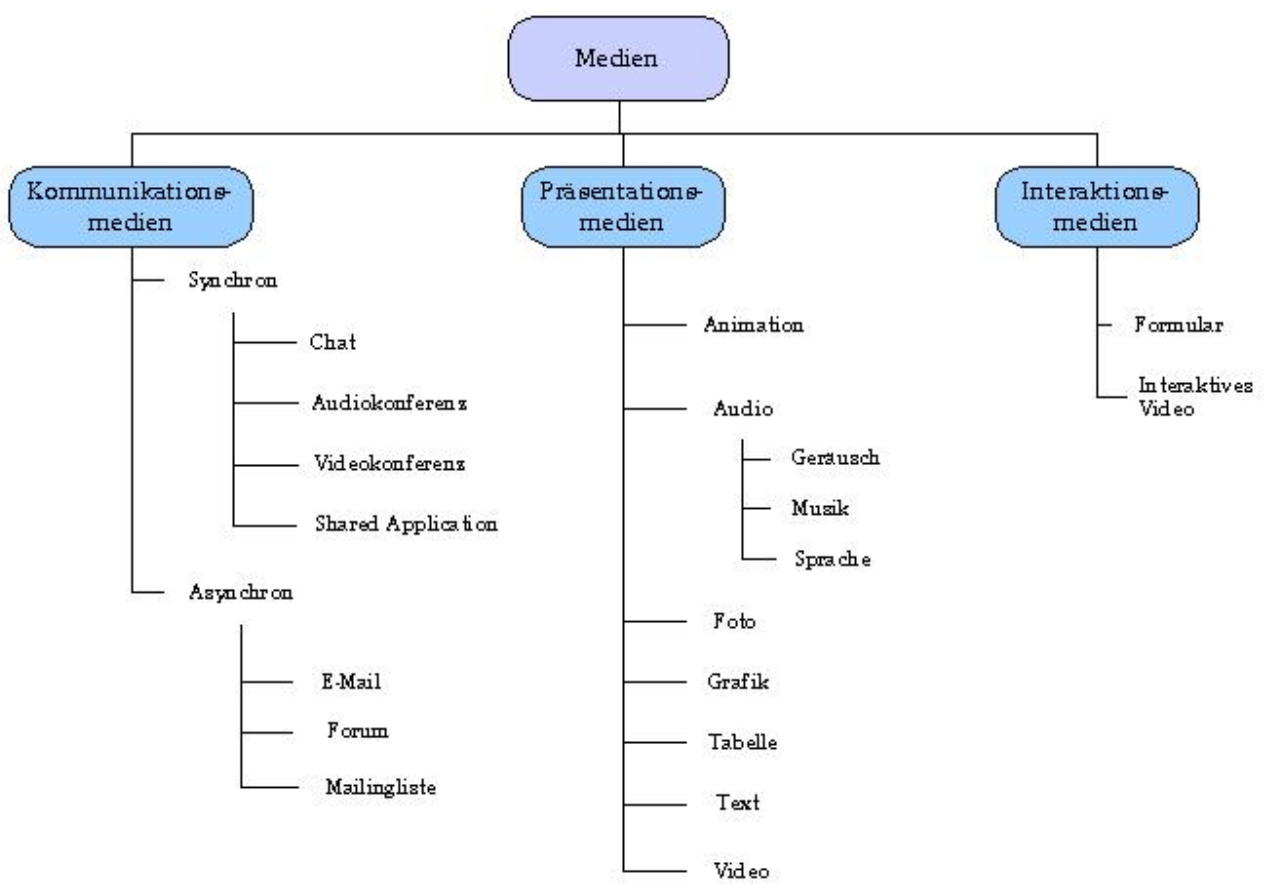

\subsubsection{Mediale Verläufe}

Die Wissenseinheiten können anhand des Medientyps in unterschiedlichen didaktischen Modellen präsentiert werden. Die Sequenzierung von Wissenseinheiten hat Meder im L-3- System als Microstrategien bezeichnet. Als mediale Microstrategien schlägt er medial beschleunigte und medial verlangsamte Verläufe vor.

- Für einen medial beschleunigten Verlauf werden die Medientypen in folgender Reihenfolge angeordnet: Text, Klang, Grafik, Foto, Video.

- Bei medial verlangsamten Verlauf werden die Medientypen in folgender Reihenfolge angeordnet: Video, Grafik, Klang, Text.

- Ergänzend zu diesen Verläufen können die Medien auch in abstrahierende und konkretisierende Verläufe angeordnet werden.

- Für einen abstrahierenden Verlauf werden die Medientypen in der Reihefolge Video, Foto, Audio, Animation, Grafik, Tabelle, Text angeordnet.

- Für einen konkretisierenden Verlauf werden die Medientypen in der Reihenfolge Text, Tabelle, Grafik, Animation, Audio, Foto, Video angeordnet. 
Die medialen Verläufe müssen dabei nicht notwendig vollständig sein. Die Reihenfolge ist so zu verstehen, dass die Medientypen in dieser Reihenfolge präsentiert werden, wenn überhaupt verschiedene Medientypen vorhanden sind. Umgekehrt läßt sich aber fordern, dass zu jeder Wissensart verschiedene Medientypen produziert werden müssen, wenn die medialen Verläufe individuell variieren sollen. Die Medientypen werden Wissensarten zugeordnet, d.h. dass eine Wissenseinheit verschiedene mediale Darstellungen einer Wissensart enthalten kann.

\subsection{Wissensarten}

Die Typisierung der Wissensarten folgt nach der L-3- Systematik von Meder wie die Medientypen den oben dargestellten Grundkategorien. Es wird zwischen rezeptivem, interaktivem und kooperativem Wissen zu unterscheiden.

\subsubsection{Rezeptive Wissenseinheiten}

Die rezeptiven Wissenseinheiten der L-3- Systematik wurden für das BIJaN-Projekt gekürzt und die Anordnung leicht überarbeitet. Die Kürzungen wurden vor allem bei Wissensarten vorgenommen, die in sozialpädagogischen Kontexten selten vorkommen (mathematische Beweise, Lehrsätze, Theoreme etc.). Einzelne Wissensarten wurden umbenannt, um die intuitive Erfassbarkeit zu verbessern. So wurde Prozedur in Anleitung umbenannt, Warum-Erklärung in Beweis und Fallerklärung in Beispiel. Die Kategorie Quellenwissen wurde erheblich gekürzt und überarbeitet. Dabei wurde auch die Kategorie Glossar gestrichen, weil ein Glossar durch eine Indexsuche nach Lerneinheiten, die Wissenseinheiten mit der Wissensart „Definition“ enthalten, erreicht werden kann. Neu hinzugefügt wurde die Kategorie „News“ unter Orientierungswissen. Damit soll insbesondere der redaktionellen Teil in BIJaN ergänzt werden. Mit der Aufnahme dieser Wissensart wird auch dem Umstand Rechnung getragen, dass die Plattform nicht in einer Webseite als Lernsystem eingebunden wird, sondern dass die Homepage ebenfalls als Lernumgebung aufgefasst wird. Die Homepage, die Selbstvorstellung etc. bilden ein Kapitel, das die Plattform und deren Organisation selbst zum Inhalt hat.

\subsubsection{Interaktive Wissenseinheiten}

Die interaktiven Wissensarten wurden aus der L-3- Systematik weitgehend übernommen. Da die BIJaN-Plattform aber auch für Online-Befragungen eingesetzt werden soll, wurde eine Kategorie für Forschungsfragen aufgenommen und mit einigen einfachen Fragetypen versehen. Diese Fragen sind als Ergänzung zu den bereits vorhandenen Fragetypen zu verstehen. Freitextfragen können z.B. in Fragebögen ebenso wie in Prüfungen verwendet werden. Lickertskalierte Fragen sind bei Tests oder Selbstkontrollaufgaben jedoch nicht gebräuchlich und wurden daher gesondert ausgewiesen. Mit der Aufnahme dieser 
Fragetypen entsteht die Möglichkeit, neben Werkzeugen für Wissenstests und Befragungen auch ein Werkzeug für die Durchführung von psychologischen Tests aufzubauen.
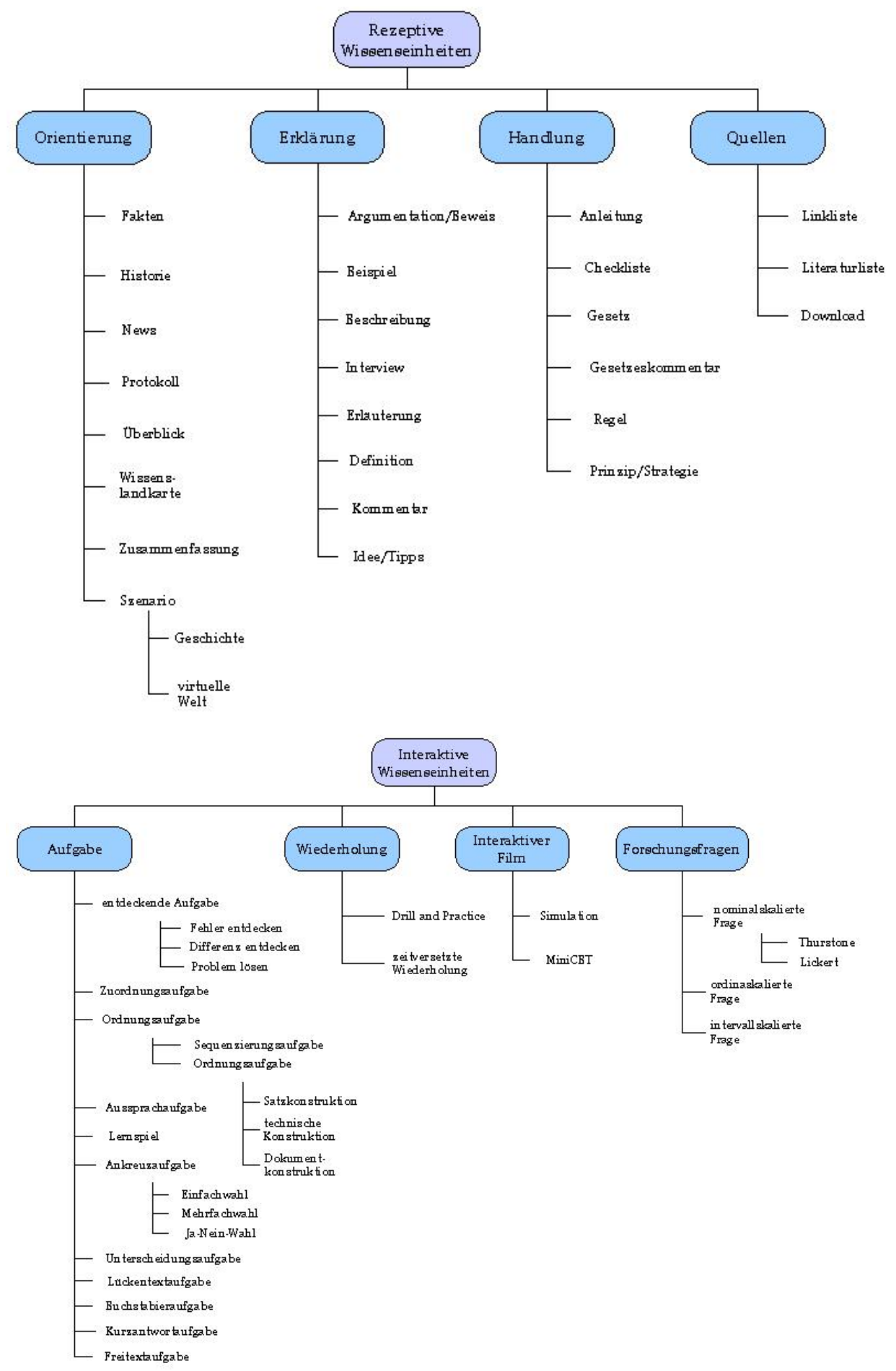


\subsubsection{Kooperative Wissenseinheiten}

Die kooperativen Wissenseinheiten sind ebenfalls weitgehend aus der L-3-Systematik von Meder übernommen. Zunächst wird zwischen geplanten und spontanen Kooperationen unterschieden. Spontane Kooperationen sind ungeplant und können jederzeit stattfinden. Geplante Kooperationen finden zu bestimmten Zeitpunkten (z.B. zur Sprechstunde) oder an einer bestimmten Stelle im Lernverlauf statt. Die Systematik der geplanten Kooperationen orientiert sich an der Gruppengröße. In Beratungen sind nur zwei Kooperationspartner anwesend, in Lerngesprächen, in Rollenspielen und Arbeitsgruppen sind kleine Gruppen (bis zu $30 \mathrm{TN}$ ) anwesend und Lernkonferenzen beziehen sich auf Großgruppen.

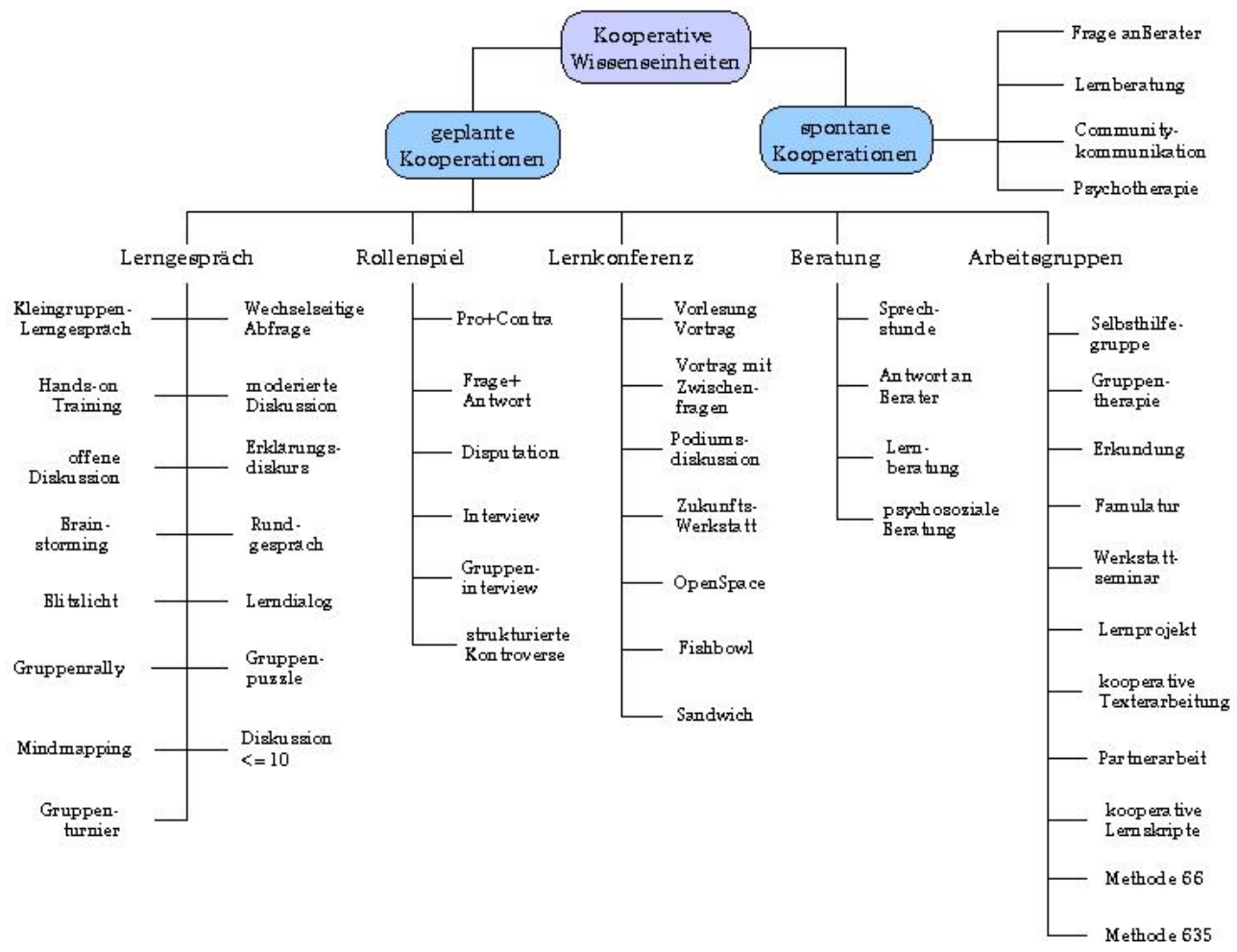

Überarbeitungen waren erforderlich, da im BIJaN eine psychosoziale Beratung und ein Communitybereich geplant sind. Um diesen Anforderungen gerecht zu werden wurde das Online-Tutoring in Beratung umbenannt. Dieser Bereich umfasst sowohl die Lernberatung als auch die psychosoziale Beratung. Die psychosoziale Beratung wurde als eigener Punkt aufgenommen, da die Zielsetzung der psychosozialen Beratung nicht mit der Zielsetzung der Lernberatung identisch ist. Gleiches gilt für die Arbeitsgruppen. Die Liste der Arbeitsgruppen wurde um Selbsthilfegruppen und Gruppentherapie ergänzt. Der Unterschied zwischen beiden Formen besteht in der Beteiligung einer bzw. eines Professionellen. Während Selbsthilfegruppen ohne professionelle Beraterinnen und Berater arbeiten und diese Rolle daher auch nicht eigens 
ausgewiesen werden muss, ist in der Gruppentherapie stets eine professionelle Beraterin oder ein professioneller Berater anwesend.

Eine ähnliche Erweiterung wurde bei den spontanen Kooperationsformen vorgesehen. Die spontane Lernberatung und die spontane psychosoziale Beratung (analog zur Telefonseelsorge) wurden unterschieden. Darüber hinaus wurde hier der Communitybereich ergänzt, der stets als spontane Kooperation aufgefasst werden kann.

Die genannten Kooperationsformen können durch eine entsprechende Medienwahl spezifiziert werden. So kann die psychosoziale Beratung mit asynchronen Medien (z.B. E-Mail) und mit synchronen Medien (z.B. Chat) durchgeführt werden. Die unterschiedlichen Rollen, z.B. in der Gruppentherapie, werden beim Anlegen der kooperativen Wissenseinheit spezifiziert. Dabei kann auch festgelegt werden, ob es sich um öffentliche oder private Kooperationsräume handelt. So können zum einen im Communitybereich private und öffentliche Kooperationsräume angelegt werden, zum anderen können etwa offene Selbsthilfegruppen oder geschlossene Therapiegruppen angelegt werden.

\subsubsection{Microverläufe}

Zwischen den Wissensarten einer Lerneinheit können didaktische Microverläufe bestimmt werden. Die Microverläufe werden aus der L-3-Systematik übernommen. Dort sind an Microverläufen geplant: Klassisch theoriegeleitet, klassisch regelgeleitet, klassisch beispielgeleitet, klassisch nach Herbart, Handlungsorientiert, aufgabenorientiertes Modell, problemorientiertes Modell, Überblickslernen, rollengeleitetes Lernen, testorientiertes Modell und das gruppenorientierte Modell.

Hier können aus Platzgründen nur zwei Microverläufe dargestellt werden. Der klassisch-theoriegeleitete Verlauf beginnt mit einer Orientierung, die angibt, worum es geht; ähnlich einem advance-organizer. Nach der Orientierung wird die Erklärung präsentiert, die sagt, warum die jeweilige Sache so ist, wie sie ist. Damit wird das theoretische Wissen zuerst präsentiert. Daran schließt sich eine Selbstkontrollaufgabe an. Nach der Selbstkontrollaufgabe wird Handlungswissen präsentiert, das sagt, was zu tun ist, wie also das begründete Wissen angewandt werden kann. Auch hieran schließt sich eine Selbstkontrollaufgabe an. Nach dem Abschlusstest wird mit Quellenwissen ein Verweis auf weitere Informationsquellen angeboten.

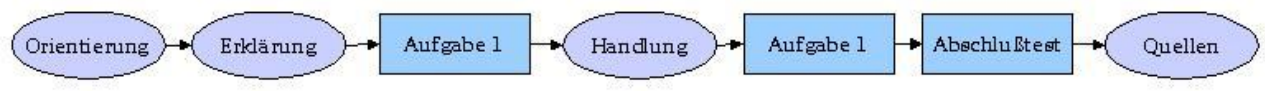

Das beispielorientierte Design gruppiert den Lernverlauf um eine Sequenz von Beispielen. Zu jedem Beispiel werden weitere Wissensarten angeboten, 
die die Lernenden je nach Bedarf abrufen können. Auch das beispielorientierte Modell wird mit einem Abschlusstest abgeschlossen.

Die Microverläufe bestimmen den didaktischen Verlauf innerhalb einer Lerneinheit. Zwischen den Lerneinheiten wird der Verlauf anhand der vorhandenen typisierten Relationen bestimmt.

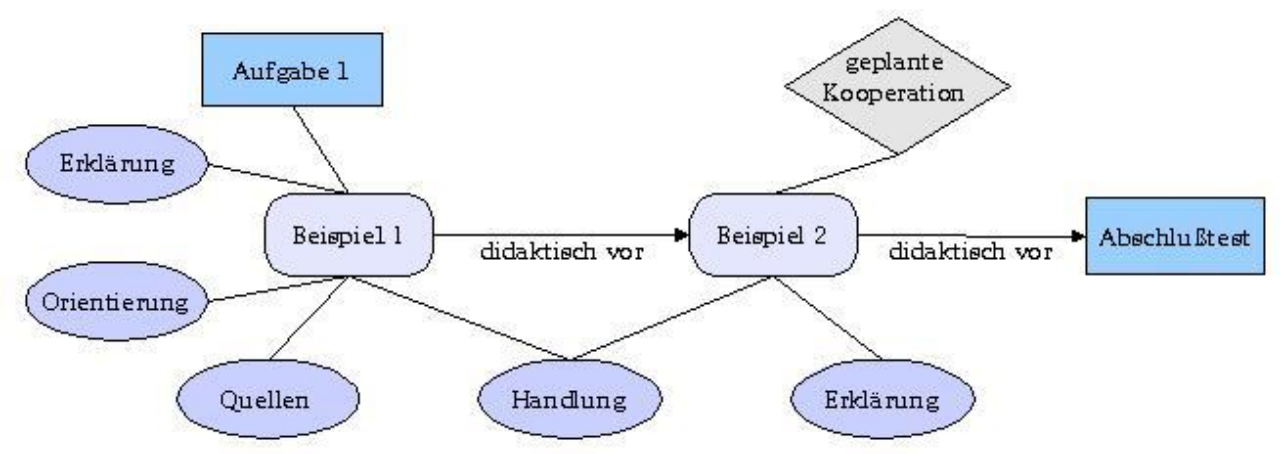

\subsection{Relationales Wissen}

Lerneinheiten umfassen ca. 5-10 Bildschirmseiten, die Wissensarten zum gleichen Thema enthalten. Die in Wissenseinheiten strukturierten Lerneinheiten werden durch typisierte Relationen miteinander verbunden. Durch das Relationensystem können verschiedene didaktische Modelle auf die Wissensbasis abgebildet werden. Diese Sequenzierung hat Meder als Macrostrategien bezeichnet.

\subsubsection{Relationstypen}

In den Relationstypen wird zunächst zwischen sachlogischen und didaktischen Relationen unterschieden. Diese Unterscheidung ist notwendig, weil es didaktische Modelle gibt, die sich nicht ausschließlich auf der sachlogischen Struktur abgebildet werden können. Die sachlogischen Relationen werden auch dafür verwendet, den Lernenden Navigationshilfen zur Aneignung des sachlogischen Kontextes eines Themas bereitzustellen. Die didaktischen Relationen können verwendet werden, um bei der Zusammenstellung von Kursen durch Lernende Themen zu identifizieren, die während der Zusammenstellung des Kurses nicht berücksichtigt wurden, die aber notwendig enthalten sein müssen, damit die recherchierten Themen angeeignet werden können.

Anhand der typisierten Relationen können Lerneinheiten automatisch in didaktische Macrostrategien angeordnet werden. 


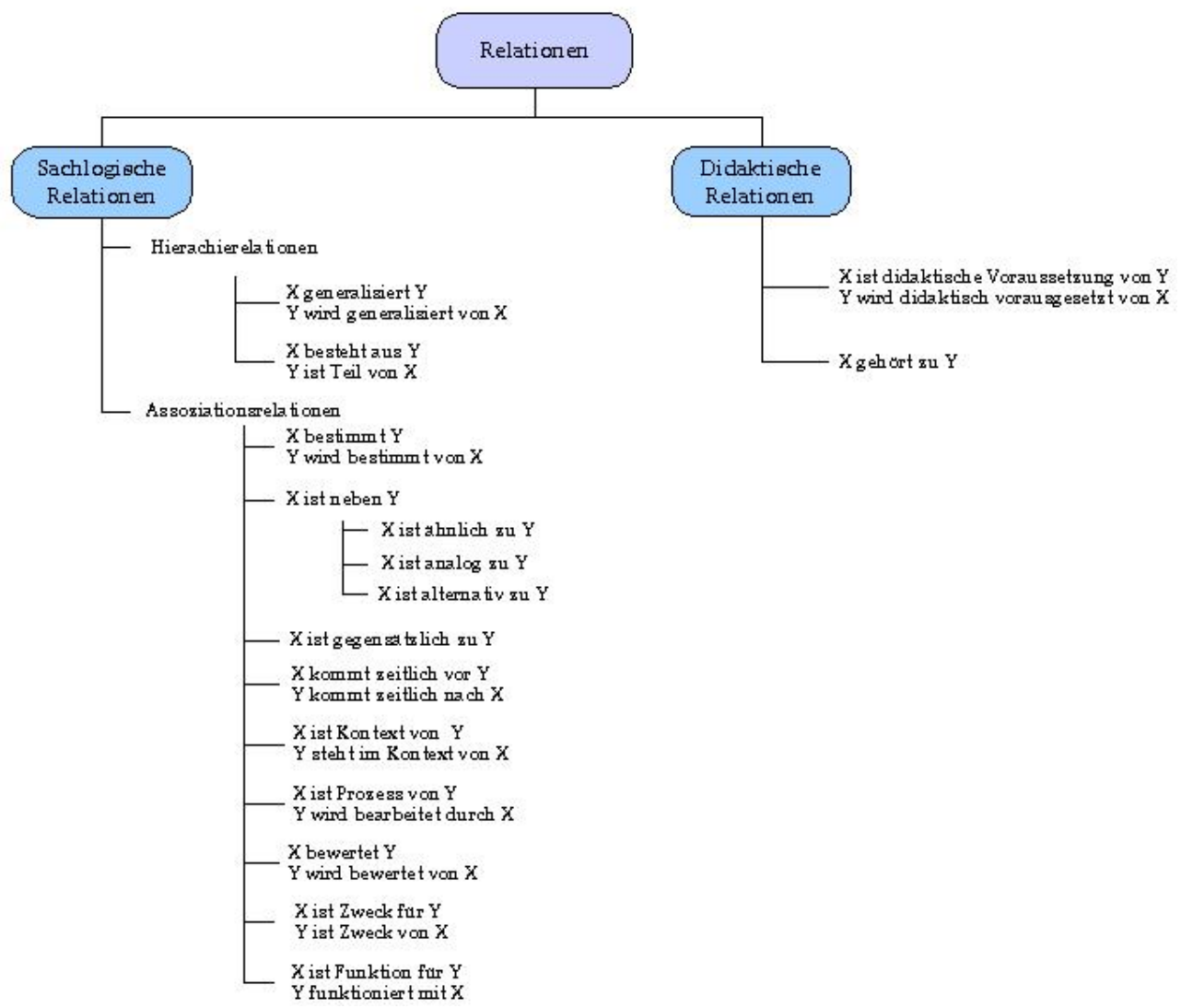

\subsubsection{Macrostrategien}

Macrostrategien beziehen sich auf die Verläufe zwischen Lerneinheiten. In den Macrostrategien wird jeweils ein Relationstyp als dominant gesetzt. Dieser Relationstyp bestimmt im wesentlichen den Lernverlauf. Weitere Relationen werden herangezogen, um in Fällen, in denen der dominante Relationstyp keine Entscheidung ermöglicht, die Sequenz zu bestimmen. Die folgenden Strategien wurden von Meder in der L-3-Systematik entwickelt:

1) Goal-based bottum up: Für dieses zielorientiert-induktive Modell werden die Lerneinheiten anhand der Hierarchierelation (generalisiert/besteht aus) angeordnet. Da dabei jede Lerneinheit mit mehreren hierarchisch höher geordneten Lerneinheiten verbunden sein kann, handelt es sich um einen Directed Acyclic Graph. Diese Struktur wird von unten nach oben zunächst so durchlaufen, daß jeweils nur das Orientierungswissen angegeben wird. Das Orientierungswissen der obersten Lerneinheit stellt das Ziel dar. Wenn dieses erreicht wird, werden die Lerneinheiten anhand der gewählten Microstrategie von unten durchlaufen. Dabei gibt es neben der Möglichkeit, während des ersten induktiven Durchlaufs alle Orientierungswissen zu präsentieren (next Top), auch die Möglichkeit, zunächst nur das Orientierungswissen der obersten Lerneinheit anzuzeigen (Top only) oder zunächst das Orientierungswissen der obersten Wissenseinheit 
anzuzeigen und anschließend die Hierarchie mit Anzeige der Orientierungswissen zu durchlaufen (Top and next Top). In Kombination mit der problemorientierten Mircostrategie realisiert diese Macrostrategie das problemorientierte Lernen. In Verbindung mit der aufgabenorienierten Microstratgie wird das aufgabenorientierte Lernen realisiert. Dabei kann zwischen wechselnden Aufgaben bei gleicher Rolle und durchgängigg gleicher Aufgabe (Lernen in virtuellen Welten) unterschieden werden.

2) Bottum up: Diese Macrostrategie beschreibt ein rein induktives Lernen. Dabei werden wie bei der zielorientierten Strategie die Lerneinheiten anhand der hierarchischen Relationen geordnet. Die unterste Lerneinheit wird identifiziert. Wenn mehrere unterste Knoten vorhanden sind, wird die erste Lerneinheit anhand der "didaktisch vor" - Relation ausgewählt. Anschließend werden die Lerneinheiten anhand der gewählten Microtragie aufsteigen durchlaufen. Dabei können entweder zunächst die auf einer Ebene liegenden Lerneinheiten nach der “didaktisch-vor” - Relation angezeigt werden, um dann in der Hierarchie aufzusteigen (Breite zuerst), oder es wird zunächst die Hierarchie bis zur obersten Lerneinheit durchlaufen, bevor die nächste Lerneinheit auf der untersten Ebene präsentiert wird.

3) Top down (deduktives Lernen): Bei dieser Macrostrategie werden die Lerneinheiten ebenfalls anhand der Hierarchierelationen angeordnet. Der oberste Knoten wird identifiziert und der Directed Acyclic Graph nach unten durchlaufen. Für die Entscheidungen bei Lerneinheiten, denen mehrere Lerneinheiten untergeordnet sind, gibt es zwei Varianten: Entweder wird der Graph erst bis zum untersten Punkt durchlaufen und dann der nächste Ast von oben begonnen, oder es werden erst die Lerneinheiten auf einer Ebene nacheinander durchlaufen und dann in die Tiefe verzweigt.

4) Das spiralmethodische Modell wird anhand der „ist Kontext von“ - Relation realisiert. Dabei kann entweder von einem Startpunkt aus immer weiter nach außen gegangen werden (dezentrativ), oder es wird ein Startpunkt außen gewählt, von dem aus dann immer weiter nach innen vorgegangen wird (konzentrativ).

5) Das aufbauende, konstruktive Modell (synthetisch) und das rekonstruktive Modell (analytisch) werden anhand der „Zweck-Mittel“ -Relation und der „bestimmt“ -Relation durchlaufen.

6) Das dialektisch nach dem Urteilsquadrat verfahrende Modell kombiniert Hierarchierelationen und Assoziationsrelationen so, daß die Lerneinheiten in der Anordnung des Urteilsquadrats der aristotelischen Logik durchlaufen werden.

7) Die Netzwerkmethode bietet die Lerneinheiten als Netzwerkstruktur an, so daß die Lernenden selbst entscheiden können, wie sie das Netzwerk durchlaufen. Dabei kann entweder eine Wissenslandkarte anhand der sachlogischen Struktur oder eine Wissenslandkarte anhand des Verlaufsnetzes eines didaktischen Modells angeboten werden.

8) Guided Tour: Bei dieser Strategie werden die Lerneinheiten anhand der Relation "ist didaktische Voraussetzung von“ sequenziert. Die Lernein- 
heiten werden also unabhängig von der sachlogischen Struktur in einer festen Abfolge angeordnet.

Es konnte noch kein Weg gefunden werden, die sokratische Methode mit Hilfe der Relationstypen abzubilden. Nur die dialektisch nach dem Urteilsquadrat verfahrende Methode, die zu den sokratischen Modellen zu rechnen ist, konnte bisher bestimmt werden. Es ist klar, dass meist nicht die benötigten Wissenseinheiten für alle hier vorgestellten didaktischen Modelle innerhalb einer Lernumgebung produziert werden können. Es ist aber mit den vorgestellten Modellen möglich, zunächst die sachlogische Struktur zu entwerfen, um didaktische Relationen zu ergänzen und dann durch eine automatische Analyse festzustellen, welche der Modelle auch tatsächlich angeboten werden können.

Umgekehrt kann eine Lernumgebung gezielt für vorher bestimmte didaktische Modelle produziert werden. Wenn z.B. aufgabenorientiert-zielorientiertes Lernen ermöglicht werden soll, ist klar, welche Wissenseinheiten für jedes Thema benötigt werden und welche Relationstypen bei der Vernetzung zu verwenden sind. Dadurch kann die didaktische Komplexität des hier vorgestellten Konzepts im Einzelfall reduziert und für Autorinnen und Autoren handhabbarer gemacht sowie institutionellen Rahmenbedingungen (z.B. Finanzierung) angepasst werden.

\subsection{Kapitel, Bücher und Büchereien}

Das hier vorgestellte Konzept zielt darauf ab, eine große Wissensbasis in kooperativer Autorenschaft zu erzeugen, in die individuelle Navigationen gelegt werden können. Dazu müssen Inhalte aus der Wissensbasis zu Kursen zusammengestellt werden. Das kann entweder durch einzelne Lernende oder durch Lehrende geschehen. In jedem Fall sind Zusammenstellungen von Medientypen zu einem Kurs erforderlich. Da im Kontext von BIJaN nicht nur Kurse, sondern auch andere Zusammenstellungen möglich sein sollen, wird eine solche Zusammenstellung als Kapitel bezeichnet. Ein Kapitel enthält Referenzen auf die zugeordneten Medientypen. Anhand der Wissenseinheiten, Lerneinheiten und Relationen, die zwischen diesen ausgewählten Medientypen bestehen, kann dann ein Lernverlauf automatisch generiert werden.

Damit diese Zusammenstellungen auch bei größeren Gebieten (bzw. längeren Kursen) noch überschaubar strukturiert werden können, ist eine Hierarchie aus Kapiteln, Büchern und Büchereien vorgesehen. Ein Kapitel kann z.B. das Material enthalten, dass an einem Tag gelernt werden kann; ein Buch enthält das Material für eine Woche und eine Bibliothek das Material für einen Monat. 


\section{Die Softwarearchitektur der Bundesinitiative Jugend ans Netz}

Die softwaretechnische Umsetzung der entwickelten Konzeption stößt zunächst auf das Problem, dass die Darstellung einzelner Wissensinhalte technisch konkretisiert werden muss. Diese technische Konkretisierung sollte nicht auf die Auswahl von Lernplattformen anhand von Qualitätskriterien beschränkt werden. Computertechnologie ermöglicht es, eine Lernplattform nicht bloß auszuwählen, sondern so zu bauen, dass sie pädagogischen Anforderungen genügt. Entsprechende pädagogische Anforderungen wurden hier vorgestellt. Die technische Umsetzung erfolgt im Rahmen des BIJaN-Projekts mit dem Applicationsserver ZOPE. ZOPE basiert auf einer objektorientierten Datenbank. Es gilt also, ein objektorientiertes Datenmodell zu entwickeln, in das die Daten gemäß der hier vorgestellten Anforderungen eingestellt werden können.

Bei der Konzeption besteht das Problem, dass die didaktische Grundstruktur zwar eine objektorientierte Darstellung nahegelegt, dass aber zugleich Relationen zwischen Objekten bestehen, die nicht mit einem objektorientierten Modell abgebildet werden können. Daher wurde als Grundstruktur eine Objekthierarchie von Lerneinheiten, Wissenseinheiten, Medientypen und Medienobjekten vorgesehen. In der untersten Ebene ist für die Mehrsprachfähigkeit der Plattform vorgesehen, Medienobjekte in verschiedenen Sprachen abzulegen. Für die typisierten Relationen zwischen Lerneinheiten und für die Zusammenstellung von Kapiteln werden Relationen verwendet, die quer zu der Objekthierarchie liegen. Mit diesem Datenmodell steht eine flexible Struktur zur Verfügung, die den am Anfang formulierten Anforderungen gerecht werden kann.

\section{Ausblick und Forschungsbedarf}

Das vorgestellte Konzept wirft eine Reihe von Forschungsfragen auf. Hier soll abschließend nur auf einige Momente hingewiesen werden, die zum Teil bereits in laufenden Studien untersucht werden:

- Eine quantitative Analyse der von Autorinnen und Autoren und Lernenden bevorzugten Lehr- und Lernstrategien anhand von Logfiles kann dazu verwendet werden, eine bessere Übereinstimmung von Lehrangebot und Lernbedarf zu erreichen. Dazu sind quantitative Verfahren zu entwickeln, die einen statistischen Vergleich von Lernverläufen ermöglichen.

- Eine quantitative Analyse der von Lernenden verwendeten Strategien in Kursen, die ohne didaktische Verläufe, d.h. nur mit Retrievalwerkzeugen angeboten werden, ermöglicht den Vergleich von empirisch gefundenen Lernstrategien mit den hier vorgestellten didaktischen Modellen. 


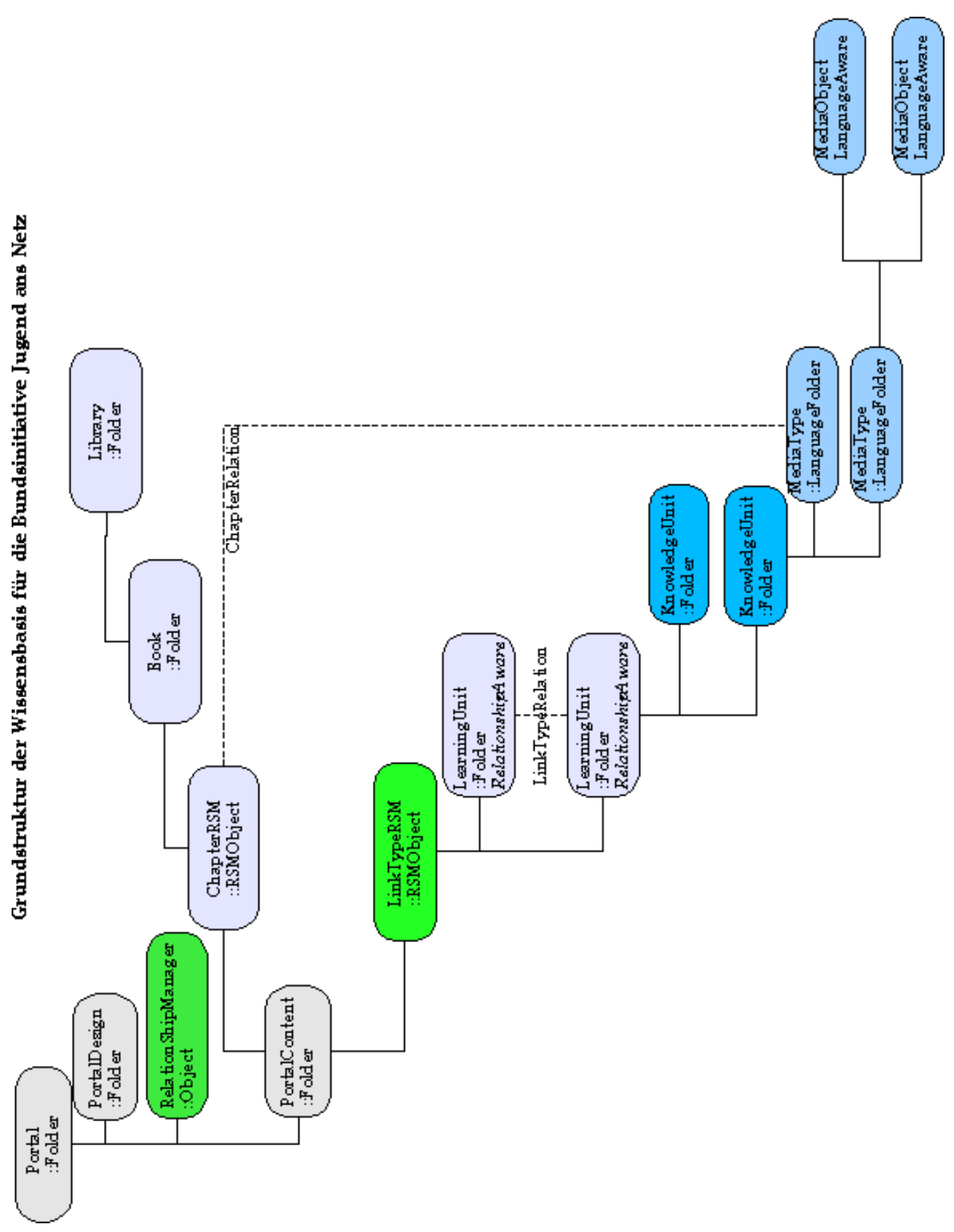


- Eine qualitative Analyse des Verhaltens der Lernenden eröffnet auf einer Mikroebene die detaillierte Beobachtung von Aneignungsprozessen. Hier sind als Varianten die Kommentierung des Lernverhaltens durch die Lernenden sowie die Interpretation des aufgezeichneten Materials durch Forscherinnen und Forscher vielversprechend.

- Die Entwicklung und Evaluation didaktischer Modelle durch Variation des Metadatensystems und der Micro- und Macrostrategien kann die Entwicklung neuer Lehrund Lernmodelle anregen.

- Um alle didaktischen Variationen in einer Lernumgebung auch tatsächlich anzubieten ist ein erheblicher Produktionsaufwand erforderlich. Es erscheint daher lohnend, empirisch zu überprüfen, welche didaktischen Verläufe von welchen Zielgruppen besonders gut angenommen werden. Diese Verläufe können dann bevorzugt produziert werden.

- Mit der Entwicklung von Online-Autorentools wird der Produktionsprozess von Lernmaterial durch Logfiles beobachtbar. Hier bietet sich die Möglichkeit, das Lehrverhalten auf einer Microebene empirisch zu untersuchen.

Für den Forschungsprozess sind durch den gemeinsamen Zugriff von verschiedenen Forschungsgruppen auf die aufgezeichneten Daten mittels Internetschnittstellen Verbesserungen in der Transparenz zu erwarten; machtförmige Forschungsstrategien, die durch Restriktionen des Zugriffs auf empirische Rohdaten möglich sind, können so von vornherein unterlaufen werden. Damit eröffnet Computertechnologie als Medium in Bildungsprozessen nicht nur neue didaktische Möglichkeiten, sondern auch interessante Forschungsperspektiven.

\section{Literatur}

Dahlberg, I.: Grundlagen universaler Wissensordnung. Pullach bei München 1974.

Döring, N.: Sozialpsychologie des Internet. Göttingen u.a. 1999.

Flechsig, K.-H.: Der Göttinger Katalog didaktischer Modelle. Nörten-Hardenberg 1983.

Hönigswald, R.: Über die Grundlagen der Pädagogik. München 1927.

Innis, $H$. A.: Tendenzen der Kommunikation. Wien, New York (Original: The Bias of Communication. Toronto 1951) 1997.

McLuhan, H. M.: Die magischen Kanäle. Understanding Media. Düsseldorf (u.a.) 1992 (Erstausgabe: ders.: Understanding Media. McGraw - Hill 1964).

Meder, N.: Prinzip und Faktum. Bonn 1975.

Meder, N.: Der Sprachspieler. Ein Bildungskonzept für die Informationsgesellschaft. In: Vjschr. f. wiss. Päd. (2) 1996, S. 145-162.

Meder, N.: Neue Technologien und Erziehung/Bildung. In: Borrelli, M./ Ruhloff, J. (Hrsg.): Deutsche Gegenwartspädagogik Bd.III, Hohengehren 1998, S. 26-40.

Meder, N.: Wissen und Bildung im Internet - in der Tiefe des semantischen Raumes. In: Marotzki, W./ Meister, D./ Sander, U. (Hrsg.): Zum Bildungswert des Internet. Opladen 2000, S. 33-56.

Meder, N.: Webdidaktik. (in Druck) 
Meyer-Drawe, K.: Menschen im Spiegel ihrer Maschinen. München 1996.

Oevermann, U.: Theoretische Skizze einer revidierten Theorie professionalisierten Handelns. In: Combe, A./ Helsper, W. (Hrsg.): Pädagogische Professionalität. Suhrkamp Wissenschaft: Frankfurt am Main 1996, S. 70-182.

Ratzek,: Zugangsverfahren. In: Buder, M./ Rehfeld, W./ Seeger, T. (Hrsg.): Grundlagen der praktischen Information und Dokumentation. München u.a. 1991, S. 232-246.

Seeger, T.: Zur Entwicklung der Information und Dokumentation. In: Buder, M./ Rehfeld, W./ Seeger, T. (Hrsg.): Grundlagen der praktischen Information und Dokumentation. München u.a. 1991, S. 9-60.

Schneider, M.: Das Urteil und die Sinne. Köln 1983.

Swertz, C.: Ausbildung zum Gebrauch didaktischer Ontologien. In: Ohly, H.P./ Rahmstorf, G./ Sigel, A. (Hrsg.): Globalisierung und Wissensorganisation. Würzburg 2000, S. 431-442.

Swertz, C.: Computer und Bildung. Bielefeld 2001 (http://archiv.ub.uni-bielefeld.de/disshabi/2001/0033/diss.ps [16.7.2002]).

Wersig, G.: Thesaurus-Leitfaden. München, New York 1978. 